\section{Einfaches Demonstrations-Flimmerphotometer}

Die technische Bewertung der Intensität verschiedenfarbiger Iichtquellen stützt sich auf die internationale Hellempfindlichkeitskurve, die ihrerseits vorwiegend auf flimmerphotometrischen Messungen beruht. Wir erlauben uns auf eine einfache Anordnung aufmerksam zu machen, die den besonderen Vorzug aufweist, da $B$ der Beobachter die beiden Lichter, die er für einen gröBeren Kreis sichtbar auf Flimmergleichheit eingestellt hat, daneben im Direktvergleich sehen kann.

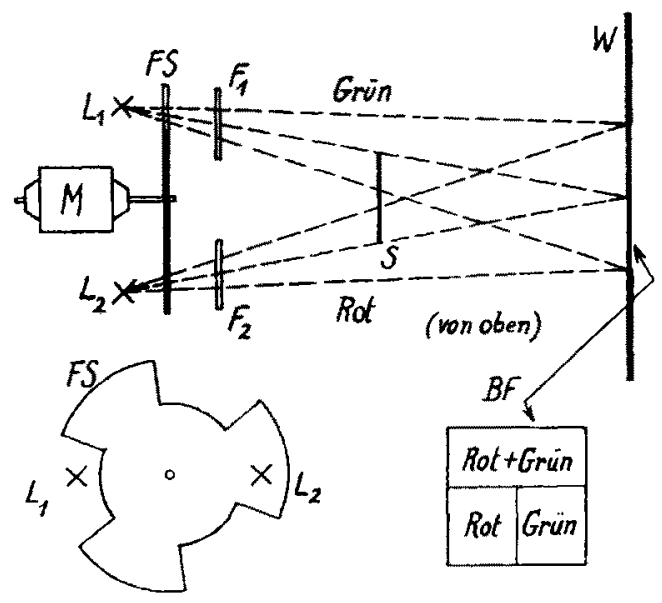

Abb. 1

Zu Abb. 1: Der Motor M treibt dic Flimmerscheibe IFS (Durchmesser: $30-50 \mathrm{~cm}$, Zahl der Lichtunterbrechungen $n$ : bis 40 pro Sekundel, welche abwechslungsweise den Weg für das Licht der Kinoprojektionslampen $\mathrm{L}_{1}$ und $\mathrm{L}_{2}$ (mindestens 200 Watt) freigibt. Die Färbung erfolgt durch Farbgläser $\left(F_{1}\right.$ und $F_{2}, z$. B. die Schott-Gläser $B G 7,1 \mathrm{~mm}$ dick, blaugrün, und $O \mathrm{OG}_{3} 3$, $1 \mathrm{~mm}$ dick, orangerot). Das Licht fällt auf eine im $\mathrm{Ab}$ stand von $1--2 \mathrm{~m}$ von der Flimmerscheibe aufgestellte, in die Lichtschutzwand W eingelassene Opalglasscheibe BF (Durchmesser $20 \mathrm{~cm}$ ). Die Beobachtung erfolgt in $\mathrm{Abb} .1$ von rechts. $\mathrm{S}$ ist ein rechteckförmiger Schirm mit einer Breite gleich dem halben Lampenabstand. Er teilt das Beobachtungsfeld $\mathrm{BF}$ in der angedeuteten Weise, so daß neben der Überlagerung die farbigen Lichter einzeln zu sehen sind. Die Veränderung der Intensität der einen Lampe erfolgt mittelst Vorwiderstand oder Reguliertransformator.

Es lassen sich unter Steigerung der Flimmerfrequenz. folgende Phänomene zeigen:

$n=O$; nach Entfernung des roten oder des grünen Glases: Simultankontrast-Wirkungen.

$n$ niedrig; oberes Feld: Sukzessivkontrast-Wirkungen.

$n$ höher, aber etwas kleiner als die optimale Flimmerfrequenz; oberes Feld a uf Flimmerminimum eingestellt: Farbflimmern verschwunden, Helligkeitsflimmern noch nicht (Prinzip des FlimmerPhotometers).

$n$ optimal; oberes Feld: in kleinem Intensitätsbereich auch Helligkeitsflimmern verschwunden (Definition der Flimmergleichheit).

n größer als die kritische Frequenz der unteren Felder: Direktvergleich der beiden im vorigen Versuch als flimmergleich erklärten Felder. $n$ wie oben, ein farbiges gegen ein ungefärbtes Licht abgeglichen: Uberschätzung der Helligkeit satter Farben (Farbenglut, "Wirksamkeitsplus").

F. Buchmülter und H. KönIG

Eidg. Amt für Maß und Gewicht, Bern, den 17. März 1945 .

\section{$-$ \\ Über die Ursache des gegensätzlichen geotropischen Verhaltens von Sproß und Wurzel}

Bekanntlich wird die Lage, welche die Organe der Pflanze im Raum einnehmen, weitgehend durch ihre Reaktion auf Licht und Schwerkraft bedingt. Fine Hauptbedeutung der. Wuchsstoffhypothese liegt nun darin, daß es mit ihrer Hilfe gelingt, die durch Schwerkraft oder Licht bewirkten Wachstumsreaktionen, also das geotropische oder phototropische Verhalten der Pflanze, auf eine gemeinsame Ursache, nämlich den Quertransport des Auxins im reagierenden Pflanzenteil zurückzuführen. WFNT ${ }^{1}$ und Dolk ${ }^{2}$ haben experimentell bewiesen, da $B$ in horizontal gelegten, also geotropisch gereizten Hafer-Koleoptilen Wuchsstoff nach unten, in einseitig belichteten, an die Schattenflanke wandert und sich dort anhäuft. Die Schwierigkeit, die einer allgemeinen Anwendung der Wuchsstoffhypothese anfänglich im Wege stand, daß nämlich nach Wuchsstolfzufuhr wohl eine Förderung des Sproßwachstums, aber stets eine Hemmung des Wurzelwachstums gefunden wurde, konnte durch den Nachweis beseitigt werden, da $B$ allgemein die Streckungswuchsstoffe je nach ihrer Konzentration das Wachstum beider Organe hemmen oder fördern, daß ihre quantitative Wirkung auf das Wachstum jedes Organs als Optimamkurve largestellt werden kann und daß zudem die Wurzel bedeutend empfindlicher auf Wuchsstoffe reagiert als der Sproß ${ }^{3,4}$. So beträgt z. B. die Wuchsstoffkonzentration für optimales Wachstum der isolierten Maiswurzel in Nährlösung bei $22^{\circ} \mathrm{C}$ nur etwa $3 \cdot 10^{-11}$ molar Heteroa uxin, für den Keimsproß der Gurke dagegen etwa $3 \cdot 10^{-4}$ molar.

Ungelöst aber ist noch immer das Problem, weshalb Sprosse in die Höhe, Wurzeln jedoch gegen den Erdmittelpunkt $z u$ wachsen, jene sich also negativ geotrop' diese sich dagegen positiv geotrop verhalten.

Aus dem tatsächlich festgestellten Empfindlichkeitsunterschied dieser Organe auf Wuchsstoff und den wachstumshemmenden respektive wachstumsfördernden Eigenschaften aller Streckungswuchsstoffe, läßt sich indes die Hypothese entwickeln, daß diese Organe $s$, und nicht anders reagieren müssen, weil normalevereise die Wurzel einen überoptimalen, der Sproß jedoch einen unteroptimalen Gehalt an Wuchsstoff besitzt. Dies bedeutet, da $B$ unter dem Einfluß der geotropischen Reizung: und infolge des Quertransportes von Wuchsstoff sich zwangslaufig auf der Unterseite der Wurzel ein stark uberoptimaler, also wachstumshemmendev, auf der Unterseite des Sprosses dagegen zwar kein optimaler, aber doch hoherer und damit gegen vorher wachstumsfördernder Gehalt an Wuchsstoff ausbilden muB.

\footnotetext{
1 WeNT, F. W., Rec. trav. bot. néerl. 25 (1928), $1-116$.

2 DoI K, H. E., Diss. Univ. Utrecht (1930).

3 Geiger-Huber, M, und Burlet, E., Jahrb. wiss, Bot. $84(1930)$,
} $233-253$.

4 Wurghitr, W. Diss. Univ. Basel (1942). 